\title{
Late complete atrioventricular block and tricuspid regurgitation after percutaneous closure of a perimembranous ventricular septal defect
}

\author{
Huiwen Chen, MD, Jinfen Liu, MD, Wei Gao, MD, and Haifa Hong, MD, Shanghai, China
}

Percutaneous closure of a perimembranous ventricular septal defect (VSD) is nowadays a valuable alternative to surgical closure. Preliminary results regarding the safety of this device and the low incidence of aortic and tricuspid regurgitation are encouraging. ${ }^{1,2}$ However, owing to the close proximity of the perimembranous VSD to the conduction system, concern about atrioventricular block has been raised. The published reports ${ }^{3}$ on this complication describe the latest onset of complete atrioventricular block (CAVB), presenting at 37.8 months after implantation. We report here 1 case of delayed CAVB with severe tricuspid regurgitation occurring 5 years after implantation of an eccentric Amplatzer perimembranous VSD occluder (APmVSDO; AGA Medical, Golden Valley, Minn).

\section{CLINICAL SUMMARY}

A $7 \frac{1}{2}$-year-old, $19-\mathrm{kg}$ boy was referred to our center for CAVB. His percutaneous procedure had been performed at 2 years 8 months in another institute. An 8-mm device was used. At presentation, he was vomiting and pale with a heart rate of 50 beats $/ \mathrm{min}$. An echocardiogram demonstrated severe tricuspid regurgitation. The ejection fraction was $64 \%$ and the fractional shortening was $34 \%$. Surgery was inevitable and performed by standard techniques of cardiopulmonary bypass, mild hypothermia, and cold blood antegrade cardioplegia. The APmVSDO was still visible transatrially (Figure 1). The tricuspid septal valve was pushed down the septum totally (Figure 1). The chordae tendineae attached to the septal and posterior valves were twisted with the APmVSDO (Figure 1). The APmVSDO was peeled off the edge of the VSD with scissors (Figure 2). The VSD (Figure 3) was $10 \mathrm{~mm}$ and closed continuously with autopericardium. The twisted chordae tendineae were refixed. The commissure of the septal and posterior valve was sutured with 2 interrupted 5-0 polypropylene sutures. An electrocardiogram still showed CAVB and a heart

\footnotetext{
From the Department of Heart Center, Shanghai Children's Medical Center, Shanghai Jiao Tong University, Shanghai, China.

Disclosures: None.

Received for publication April 11, 2010; accepted for publication May 14, 2010; available ahead of print July 20, 2010.

Address for reprints: Jinfen Liu, MD, Department of Heart Center, Shanghai Children's Medical Center, Shanghai Jiao Tong University, Shanghai, China 862138626161-3001 (E-mail: scmcmud@online.sh.cn).

J Thorac Cardiovasc Surg 2010;140:e60-1

$0022-5223 / \$ 36.00$

Copyright (c) 2010 by The American Association for Thoracic Surgery doi:10.1016/j.jtcvs.2010.05.025
}

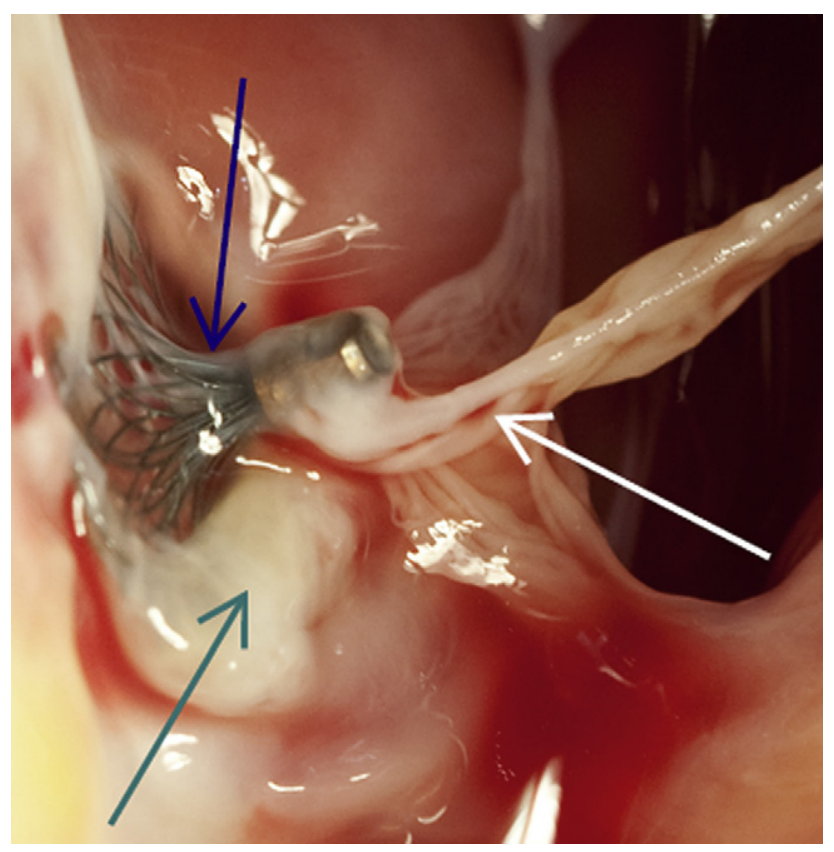

FIGURE 1. Amplatzer perimembranous ventricular septal defect occluder (APmVSDO) (blue arrow) was visible transatrially, the tricuspid septal valve (green arrow) was pushed down the septum totally, and the chordae tendineae (white arrow) attached to the septal and posterior valves were twisted with the APmVSDO.

rate of 50 to 60 beats/min. Transesophageal echocardiography showed no residual shunt and trivial tricuspid regurgitation. The heart rate was 50 to 60 beats/min after 2 weeks' monitoring in the hospital. He underwent pacemaker implantation. An echocardiogram performed at discharge showed trivial tricuspid regurgitation, an ejection fraction of $72 \%$, and a fractional shortening of $41 \%$. Outpatient follow-up was advised with an electrocardiogram and echocardiogram at 1 week, 1 month, 6 months, 1 year, and then yearly.

\section{DISCUSSION}

Preliminary results of transcatheter closure of perimembranous VSD by an APmVSDO are encouraging, with no mortality, high short-term technical success, and high occlusion rates in the absence of any major concerns. ${ }^{3}$ The only serious concern is the occurrence of CAVB. Butera and colleagues ${ }^{4}$ report longer term follow-up in 104 patients at a median of 38.5 months. The incidence of 


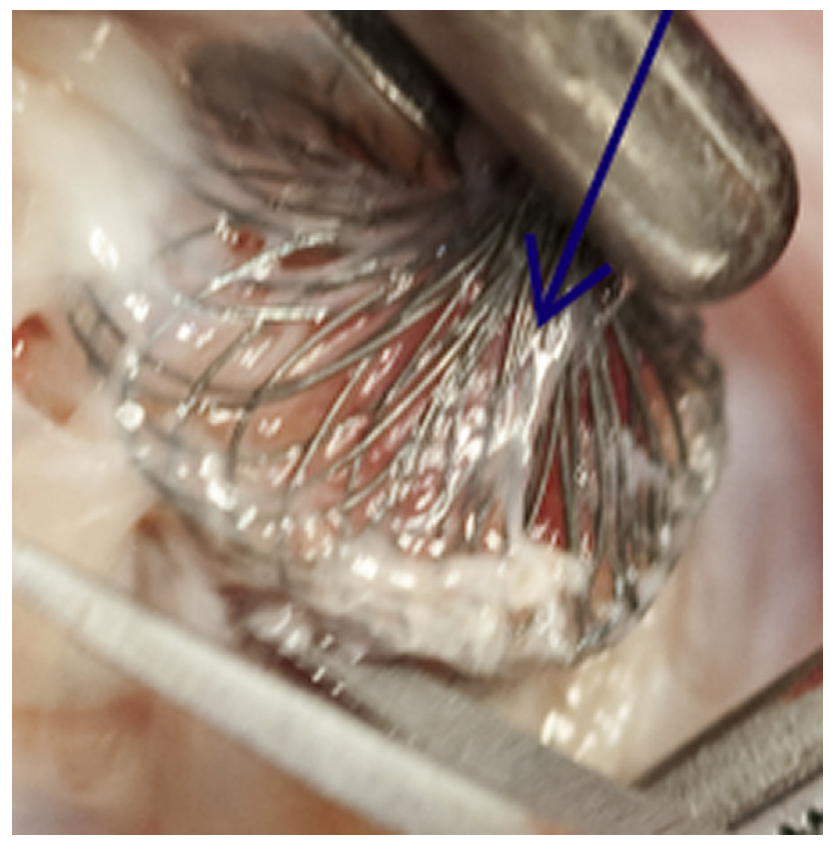

FIGURE 2. The Amplatzer perimembranous ventricular septal defect occluder (APmVSDO) (blue arrow) was peeled off the edge of ventricular septal defect with scissors.

complete heart block was $8.7 \%$, and a permanent pacemaker was required in $6(5.7 \%)$ patients, 2 in the early phase and 4 during late follow-up. Predescu and coworkers ${ }^{3}$ reported that $4(22 \%)$ had complete heart block at 17 days, 4.2 months, 8.8 months, and 37.8 months after implantation, respectively. On the basis of these data, implantation of the perimembranous VSD device was terminated at Predescu's institution (the Hospital for Sick Children, Toronto, Ontario, Canada). To our knowledge, this is the first study reporting the latest occurrence of CAVB with severe tricuspid regurgitation in patients treated percutaneously for a perimembranous VSD.

It is possible that the right ventricular disc impinging on the septal and posterior leaflet and the device shift may cause it to disturb atrioventricular conduction by direct traumatic compression. Atrioventricular conduction tissue trauma and tricuspid regurgitation led to a vicious circle of further trouble. Increased tricuspid regurgitation may be a risk factor of CAVB during short- and medium-term follow-up.

Surgical intervention for isolated perimembranous VSD in experienced centers has achieved this with minimal mortality and a very low incidence of CAVB.

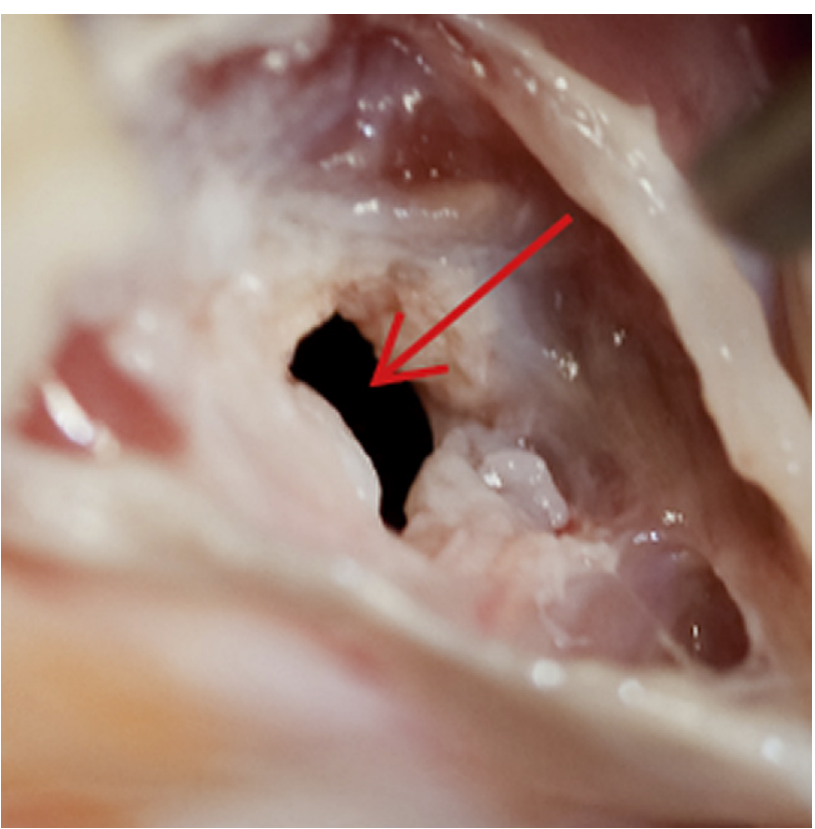

FIGURE 3. The ventricular septal defect (red arrow) after removal of the Amplatzer perimembranous ventricular septal defect occluder (APmVSDO).

Tucker and associates ${ }^{5}$ reported that the incidence of permanent pacemaker implantation was $0.4 \%$ and hospital mortality was $0.2 \%$ in the contemporary era of 1315 patients undergoing repair. Patients and their parents should be informed to make a choice between the morbidity of cardiopulmonary bypass and the risk of sudden, potentially lifethreatening late-onset CAVB.

\section{References}

1. Hijazi ZM, Hakim F, Haweleh AA, Madani A, Tarawna W, Hiari A, et al. Catheter closure of perimembranous ventricular septal defects using the new Amplatzer membranous VSD occluder: initial clinical experience. Catheter Cardiovasc Interv. 2002;56:508-15.

2. Bass JL, Kalra GS, Arora R, Masura J, Gavora P, Thanopoulos BD, Torres W, Sievert H, Carminati M, Fischer G, Ewert P. Initial human experience with the Amplatzer perimembranous ventricular septal occluder device. Catheter Cardiovasc Interv. 2003;58:238-45.

3. Predescu D, Chaturvedi RR, Friedberg MK, Benson LN, Ozawa A, Lee KJ. Complete heart block associated with device closure of perimembranous ventricular septal defects. J Thorac Cardiovasc Surg. 2008;136:1223-8.

4. Butera G, Carminati M, Chessa M, Piazza L, Micheletti A, Negura DG, et al. Transcatheter closure of perimembranous ventricular septal defects-early and long-term results. J Am Coll Cardiol. 2007;50:1189-95.

5. Tucker EM, Pyles LA, Bass JL, Moller JH. Permanent pacemaker for atrioventricular conduction block after operative repair of perimembranous ventricular septal defect. J Am Coll Cardiol. 2007;50:1196-200. 